\title{
Willingness to Attend Sex Venues in the Context of the COVID-19 Pandemic in New York City: Results from an Online Survey with Sexual and Gender Minority Individuals
}

\author{
Étienne Meunier (D) Anne E. Sundelson - Stephen Tellone • Daniel Alohan • \\ Celia B. Fisher • Christian Grov
}

Accepted: 25 March 2021/ Published online: 4 May 2021

(C) The New York Academy of Medicine 2021

\begin{abstract}
Collective sex venues (places where people have sex in groups or in the presence of others, such as bathhouses or sex clubs) are locations where SARS-CoV2 transmission is likely to occur. We conducted an online survey to examine the impact of the COVID-19 pandemic among 342 sexual and gender minority (SGM) individuals who had attended collective sex venues (CSV) in New York City (NYC) in the prior year. Almost 1 in 10 (9.9\%) participants reported having received a positive test for SARS-CoV-2 infection or antibodies. Although a minority (27.5\%) of participants reported being comfortable attending a CSV during the COVID-19 pandemic, multivariable ordinal logistic regression found that willingness was higher among participants who had taken the survey later in the pandemic $\left(\mathrm{aOR}=2.90, \mathrm{CI}_{95 \%} 1.90\right.$ to 4.43$)$, who attended $\mathrm{CSV}$ at higher frequencies $\left(\mathrm{aOR}=1.94, \mathrm{Cl}_{95 \%}\right.$ 1.26 to 2.99), who used substances at $\mathrm{CSV}(\mathrm{aOR}=1.98$, $\mathrm{CI}_{95 \%} 1.22$ to 3.23 ), and who had tested positive for SARS-CoV-2 infection or antibodies $\left(\mathrm{aOR}=2.27, \mathrm{CI}_{95 \%}\right.$
\end{abstract}

É. Meunier $(\bowtie) \cdot$ A. E. Sundelson $\cdot$ S. Tellone $\cdot$ D. Alohan Department of Sociomedical Sciences, Columbia University Mailman School of Public Health, 722 West 168th Street 9th Fl., New York, NY 10032, USA

e-mail: e.meunier@columbia.edu

C. B. Fisher

Center for Ethics Education and Department of Psychology, Fordham University, New York, NY, USA

C. Grov

Department of Community Health and Social Sciences, Graduate School of Public Health and Health Policy, City University of New York, New York, NY, USA
1.17 to 4.39 ). In open survey answers, participants described reasons for or against attending CSV during the pandemic, as well as risk reduction strategies that would make them more comfortable attending (e.g., screening for test results, doing temperature checks, holding outdoor events, or restricting events to lower risk sexual practices). SGM individuals who attend CSV might be at increased risk for COVID-19. Public health officials should provide CSV organizers and attendees with guidelines on how to prevent or minimize transmission risk in the context of pandemics such as COVID-19.

Keywords Sexual and gender minority individuals . Collective sex $\cdot$ SARS-CoV-2 $\cdot$ COVID-19 $\cdot$ Sexual behaviors

\section{Introduction}

First observed in China in December 2019, the severe acute respiratory syndrome coronavirus 2 (SARS-CoV2) rapidly spread around the world, leading to the global COVID-19 pandemic. In spring 2020, New York City (NYC) became a global epicenter of the pandemic - its first case was confirmed on March 1, 2020, and there were 21,000 total confirmed cases of COVID-19 in the city by March $23[1,2]$. In an attempt to quell the exponential growth of cases and deaths, state and city government officials put in place a series of restrictions closing non-essential businesses and schools and requiring non-essential gatherings to be canceled [3]. In May, following a decrease of daily new cases, NYC began a 
phased reopening, allowing for gatherings of up to 10 people and for businesses such as restaurants to reopen, provided they enforce proper social distancing guidelines. However, NYC saw a resurgence of COVID-19 cases in November and December 2020 [4], and Mayor Bill de Blasio has warned New Yorkers about the potential for a second citywide shutdown.

Because physical proximity and respiratory droplets are important routes of transmission of SARS-CoV-2, the virus is likely to spread during sexual activity [5]. To minimize the risk of transmission between sex partners during the pandemic, public health officials, including the NYC Department of Health and Mental Hygiene (DoHMH), have advised people to avoid or limit sexual contact with individuals outside of their household [6]. Researchers have suggested that it may be particularly challenging for sexual and gender minority (SGM) individuals to adhere to these social distancing guidelines as they often rely on their social networks and sexual partners for support [7, 8]. Although some studies have found that sexual minority men (SMM) have reported significantly fewer sex partners since the start of the pandemic [9-12], others have documented increases in sexual activity among SMM $[13,14]$. Specifically, studies have found that SMM who, prior to the pandemic, engaged in group sex, sexualized drug use (chemsex), or sex work have reported similar or increased sexual activity during the pandemic $[10,13,14]$.

As physical proximity is an important factor in the transmission of SARS-CoV-2, attendees of collective sex venues (e.g., bathhouses, sex clubs, or sex parties) could be at high risk for COVID-19. Research examining HIV/STI risk in such venues has found that attendees often have multiple sex partners during one visit [15-18], and ethnographic data has revealed that sex clubs and sex parties often bring together large numbers of attendees in one crowded space for sex [19-21]. Although commercial sex venues would theoretically have to follow the same lockdown restrictions affecting bars and nightclubs, in NYC, collective sex usually takes place in clandestine private sex clubs or sex parties due to state regulations prohibiting sex in commercial establishments [22]. Since March 2020, the NYC DoHMH has advised local sex party organizers to discontinue their activities due to the pandemic, and many seem to have complied [23]. However, some media accounts have claimed that NYC's sex scene is still active [24-26]. Ultimately, the decision rests with individuals regarding hosting or attending sex venues amid the COVID-19 pandemic and, if so, what protections to take to minimize the risk of SARS-CoV-2 transmission.

Considering the elevated risk of transmission of respiratory viruses such as SARS-CoV-2 in collective sex venues, more needs to be learned about the reasons why some individuals are willing to attend such venues in the context of a pandemic. To address this issue, we examined survey data collected during the COVID-19 pandemic among SGM individuals from NYC who had attended collective sex venues in the prior year.

\section{Methods}

Data reported here were collected as part of a study examining the acceptability of sexual health promotion interventions at collective sex venues in NYC. The study had been designed prior to the pandemic but, as data collection was planned for the spring of 2020, items were added to examine the impact of COVID-19 on attendees of these venues. Participants were recruited via online advertisements on social media, hookup apps (smartphone applications used to meet romantic or sex partners), and emails sent by local sex party promoters. Advertisements invited individuals who had attended group sex venues/events to take a screener questionnaire of no more than $5 \mathrm{~min}$ to see if they were eligible. To be included, participants had to (a) be at least 18 years old; (b) report living in the NYC Metropolitan Area on March 1, 2020; (c) identify as a cisgender man or as a transgender or nonbinary individual; (d) report having had sex with a male partner in the prior year; and (e) report having had sex, in the prior 12 months, in a collective sex venue (defined as a bathhouse, bar/nightclub, sex club, sex party, or adult video store/theater).

Screening and data collection procedures took place between May 9 and September 23, 2020. We emailed a unique, single-use weblink to eligible screener respondents inviting them to complete the online survey for the study (of approximately $20 \mathrm{~min}$ ), for which they would earn a \$10 electronic Amazon.com gift card. The survey gathered information about demographics, sexual behaviors, sexual health, substance use, collective sex venue attendance, and attitude towards sexual health promotion at sex venues. Participants were also asked if they had ever taken a test for SARS-CoV-2 infection or antibodies, whether they had ever received a positive result, and how worried they were about getting infected or reinfected with the virus on a scale of 1 (not worried 
at all) to 4 (extremely worried). We also asked how comfortable participants would be attending a sex venue in the context of the COVID-19 pandemic on a scale of 1 (extremely uncomfortable) to 5 (extremely comfortable). We then asked participants if they had engaged in a series of social and sexual behaviors since March 15 , 2020, and which, among a series of statements, would make them more comfortable attending sex venues in the context of the pandemic (listed in Table 2). Finally, an open-text question asked participants to share anything else that affected their willingness to attend sex venues in the context of the COVID-19 pandemic. The Institutional Review Board at Columbia University approved the protocol.

Statistical analyses were conducted using IBM SPSS Statistics version 26 . We examined bivariate associations between comfort attending a sex venue in the context of the COVID-19 pandemic and a series of demographic and behavioral variables using the Kruskal-Wallis test for categorical variables and ordinal regressions for continuous variables. We then conducted multivariable ordinal regressions to identify associations between comfort attending sex venues in the context of the pandemic and the variables found to be significantly associated with it in bivariate analyses. We used descriptive statistics to report on social/sexual behaviors since the start of the pandemic and the factors that would make participants more comfortable attending sex venues. Finally, we did a qualitative analysis of the open-text answers using the software Dedoose. In the first round of coding, the first two authors read and categorized participants' answers to develop a coding guide. They then collaboratively applied the coding guide to every response and discussed differences to resolve them. Then, the third and fourth authors were asked to independently apply the coding guide to a sample of responses to assess reliability. Coding discrepancies were discussed among the team until agreement was achieved on the categorization of the data, presented in Table 4.

\section{Results}

A total of 724 potential participants completed the screener questionnaire and provided contact information to receive the full survey. Almost two-thirds of them $(65.5 \%)$ reported having heard about the study from a sex party promoter, $19.1 \%$ from an advertisement on a hookup app, $8.4 \%$ on social media, and $6.5 \%$ from someone who told them about it. There were $498(68.8 \%)$ respondents who were determined to be eligible for the study and were sent a link to complete the full survey. Of them, 359 $(72.1 \%)$ completed the survey but 17 were determined ineligible based on their answers, yielding an analytic sample of 342 . As recruitment activities were done in two waves, $54.1 \%$ of participants completed the survey between May 24 and July 8, and 45.9\% did so between August 8 and September 23, 2020.

\section{Participant Characteristics}

Table 1 provides descriptive statistics for the final analytic sample $(n=342)$. Age ranged from 18 to 72 years old (mean: 36.4; median: 34). Most (90.6\%) participants identified as cisgender men, the others identifying as transgender men $(2.0 \%)$, transgender women $(0.9 \%)$, or gender non-conforming $(6.5 \%)$. White, non-Hispanic participants represented $59.1 \%$ of the sample. The majority were single $(62.6 \%)$ and identified as gay, queer, or pansexual $(94.2 \%)$. The socio-economic status of the sample was relatively high as only $14.9 \%$ had not earned a bachelor's degree and only $33.4 \%$ reported income of less than $\$ 50,000$ a year. Fifty-eight respondents $(17.0 \%)$ reported having been diagnosed with HIV. Of those who reported never receiving a positive diagnosis for HIV, most (70.8\%) reported currently using pre-exposure prophylaxis ( $\mathrm{PrEP})$.

Over half of the sample $(53.8 \%)$ reported having attended sex venues 5 times or more in the prior year, while the remaining $(46.2 \%)$ had done so between 1 and 4 times. The proportion of participants who reported consuming alcohol during or shortly before their sex venue visits in the prior year was $59.6 \% ; 58.2 \%$ reported using poppers, $34.2 \%$ erectile dysfunction (ED) drugs, $30.1 \%$ marijuana, $10.8 \%$ GHB, $10.2 \%$ MDMA, $9.4 \%$ ketamine (special $\mathrm{K}$ ), $9.1 \%$ powdered cocaine, $6.4 \%$ crystal methamphetamine, and $2.3 \%$ LSD (participants reported no other substances used at sex venues). To avoid multicollinearity in the multivariable analysis, we created a variable measuring the use of any "hard drug" (GHB, MDMA, special K, cocaine, crystal meth, or LSD) $-23.1 \%$ of participants reported using any one of these substances at a sex venue in the prior year. 
Table 1 Participant characteristics and comfort attending sex venues

\begin{tabular}{|c|c|c|c|c|c|}
\hline & \multicolumn{2}{|c|}{ Total } & \multicolumn{3}{|c|}{ Comfort going to sex venues } \\
\hline & $n$ & $\%$ & Mean $^{\mathrm{a}}$ & $\mathrm{K}-\mathrm{W} H^{\mathrm{b}}$ & $p$ value $^{\mathrm{c}}$ \\
\hline Total sample & 342 & $(100.0)$ & 2.37 & & \\
\hline Date survey taken (2021) & & & & 26.047 & $<.001$ \\
\hline May 24 to July 8 & 185 & $(54.1)$ & 2.03 & & \\
\hline August 8 to Sept 23 & 157 & $(45.9)$ & 2.76 & & \\
\hline Age (median; IQR) & 34 & $29-41$ & & 0.58 & .446 \\
\hline Race/ethnicity & & & & 5.916 & .206 \\
\hline Asian & 32 & $(9.4)$ & 1.91 & & \\
\hline Black & 36 & $(10.5)$ & 2.56 & & \\
\hline Latino, any race & 55 & $(16.1)$ & 2.44 & & \\
\hline Multiracial/Other & 17 & $(5.0)$ & 2.47 & & \\
\hline White & 202 & $(59.1)$ & 2.38 & & \\
\hline Sex/gender & & & & 4.917 & .027 \\
\hline Cisgender man & 310 & $(90.6)$ & 2.42 & & \\
\hline Transgender/nonconforming & 32 & $(9.4)$ & 1.88 & & \\
\hline Relationship & & & & 0.524 & .469 \\
\hline Single & 214 & $(62.6)$ & 2.39 & & \\
\hline In a relationship & 128 & $(37.4)$ & 2.33 & & \\
\hline Sexual identity & & & & 0.009 & .924 \\
\hline Gay, queer, pansexual & 322 & $(94.2)$ & 2.36 & & \\
\hline Bisexual, heterosexual & 20 & $(5.8)$ & 2.45 & & \\
\hline Education & & & & 0.898 & .638 \\
\hline Less than bachelor's & 51 & (14.9) & 2.47 & & \\
\hline Bachelor's & 155 & $(45.3)$ & 2.3 & & \\
\hline Graduate & 136 & $(39.8)$ & 2.4 & & \\
\hline Income/year & & & & 0.586 & .746 \\
\hline Less than $\$ 50 \mathrm{~K}$ & 112 & $(33.4)$ & 2.31 & & \\
\hline$\$ 50 \mathrm{~K}$ to $\$ 99.9 \mathrm{~K}$ & 127 & $(37.9)$ & 2.42 & & \\
\hline$\$ 100 \mathrm{~K}$ or more & 96 & $(28.7)$ & 2.31 & & \\
\hline HIV status/PrEP use & & & & 3.388 & .184 \\
\hline Negative, not using PrEP & 83 & $(24.3)$ & 2.14 & & \\
\hline Negative, using PrEP & 201 & $(58.8)$ & 2.4 & & \\
\hline Positive & 58 & $(17.0)$ & 2.55 & & \\
\hline Sex venue attendance, $\mathrm{PY}$ & & & & 8.116 & .004 \\
\hline 1 to 4 times & 158 & $(46.2)$ & 2.12 & & \\
\hline 5 times or more & 184 & $(53.8)$ & 2.58 & & \\
\hline CV-19 tested ${ }^{\mathrm{c}}$ & & & & 8.371 & .004 \\
\hline Never & 160 & $(46.8)$ & 2.14 & & \\
\hline Yes & 182 & $(53.2)$ & 2.56 & & \\
\hline Tested positive for $\mathrm{CV}-19^{\mathrm{d}}$ & & & & 14.421 & $<.001$ \\
\hline Never & 308 & $(90.1)$ & 2.27 & & \\
\hline Yes & 34 & $(9.9)$ & 3.24 & & \\
\hline Worried about CV-19 & & & & 49.502 & $<.001$ \\
\hline A little or not & 215 & $(62.9)$ & 2.74 & & \\
\hline
\end{tabular}


Table 1 (continued)

\begin{tabular}{|c|c|c|c|c|c|}
\hline & \multicolumn{2}{|c|}{ Total } & \multicolumn{3}{|c|}{ Comfort going to sex venues } \\
\hline & $n$ & $\%$ & Mean $^{\mathrm{a}}$ & $\mathrm{K}-\mathrm{W} H^{\mathrm{b}}$ & $p$ value ${ }^{\mathrm{c}}$ \\
\hline Quite or extremely & 127 & $(37.1)$ & 1.72 & & \\
\hline Used alcohol at CSV, PY & & & & 0.541 & .462 \\
\hline Yes & 204 & $(59.6)$ & 2.31 & & \\
\hline No & 138 & $(40.4)$ & 2.44 & & \\
\hline Used marijuana at CSV, PY & & & & 0.127 & .722 \\
\hline Yes & 103 & $(30.1)$ & 2.32 & & \\
\hline No & 239 & $(69.9)$ & 2.38 & & \\
\hline Used poppers at CSV, PY & & & & 5.735 & .017 \\
\hline Yes & 199 & $(58.2)$ & 2.5 & & \\
\hline No & 143 & $(41.8)$ & 2.17 & & \\
\hline Used EDD at CSV, PY & & & & 7.946 & .005 \\
\hline Yes & 117 & $(34.2)$ & 2.67 & & \\
\hline No & 225 & $(65.8)$ & 2.21 & & \\
\hline Used GHB at CSV, PY & & & & 19.892 & $<.001$ \\
\hline Yes & 37 & $(10.8)$ & 3.32 & & \\
\hline No & 305 & $(89.2)$ & 2.25 & & \\
\hline Used crystal at CSV, PY & & & & 13.397 & $<.001$ \\
\hline Yes & 22 & $(6.4)$ & 3.36 & & \\
\hline No & 320 & $(93.6)$ & 2.3 & & \\
\hline Used powder cocaine at CSV, PY & & & & 1.246 & .264 \\
\hline Yes & 31 & $(9.1)$ & 2.61 & & \\
\hline No & 311 & $(90.9)$ & 2.34 & & \\
\hline Used MDMA at CSV, PY & & & & 3.915 & .048 \\
\hline Yes & 35 & $(10.2)$ & 2.8 & & \\
\hline No & 307 & $(89.8)$ & 2.32 & & \\
\hline Used special $\mathrm{K}$ at $\mathrm{CSV}, \mathrm{PY}$ & & & & 8.151 & .004 \\
\hline Yes & 32 & $(9.4)$ & 3.03 & & \\
\hline No & 310 & $(90.6)$ & 2.3 & & \\
\hline Used LSD/acid at CSV, PY & & & & 0.062 & .804 \\
\hline Yes & 8 & $(2.3)$ & 2.5 & & \\
\hline No & 334 & $(97.7)$ & 2.36 & & \\
\hline Any hard drug use at CSV, PY & & & & 17.86 & $<.001$ \\
\hline No & 263 & $(76.9)$ & 2.2 & & \\
\hline Yes & 79 & $(23.1)$ & 2.92 & & \\
\hline
\end{tabular}

${ }^{\mathrm{a}}$ Average score of responses where $1=$ extremely uncomfortable, $2=$ somewhat uncomfortable, $3=$ neither comfortable nor uncomfortable, $4=$ somewhat comfortable, and $5=$ extremely comfortable

${ }^{\mathrm{b}}$ This column reports Kruskal-Wallis $H$ statistic except for one continuous variable (age), which reports Pearson's $\chi^{2}$ statistic

${ }^{\mathrm{c}}$ Bolded values indicate significance at the $\alpha=.05$ level

${ }^{\mathrm{d}}$ Antibodies or current infection

${ }^{\mathrm{e}}$ Participants who reported using any one of the following substances at collective sex venues in the prior year: GHB, MDMA, special K, cocaine, crystal meth, or LSD

$I Q R$, interquartile range; $C S V$, collective sex venue; $P Y$, past year; $C V-19$, COVID-19/SARS-CoV-2; $E D D$, erectile dysfunction drugs 


\section{COVID-19}

A little over half (53.2\%) of participants reported having ever taken a test for SARS-CoV-2, either for antibodies or current infection. Among the whole sample, 9.9\% reported ever receiving a positive diagnosis (either for SARS-CoV-2 antibodies or current infection). Most (62.9\%) participants reported being not worried or only a little worried about getting COVID-19, the others feeling quite or extremely worried.

Participants reported on their social and sexual behaviors between March 15, 2020, and the time they took the survey (Table 2). Over two-thirds (68.7\%) reported that they had sex with a partner they did not live with during that timeframe, and $57.3 \%$ reported having met new sex partners online. A little over a third (35.4\%) reported having engaged in group sex (i.e., three partners or more), or attended an in-person social event $(34.8 \%)$.

When asked how comfortable they would be attending sex venues in the context of the COVID-19 pandemic, $37.1 \%$ said "extremely uncomfortable (1)," $24.3 \%$ "somewhat uncomfortable (2)," $11.1 \%$ "neither comfortable nor uncomfortable (3)," $19.9 \%$ "somewhat comfortable (4)," and 7.6\% "extremely comfortable (5)"; the mean level of comfort was 2.37. From most to least endorsed, the factors that would increase participants' willingness to go back to sex venues were (Table 2) "if a vaccine was developed" (81.0\%), "if the number of daily infections decreased significantly" $(61.7 \%)$, "if sex venues screened for fever at entry" (55.8\%), "if I tested positive for antibodies" (53.8\%), "if authorities allowed bars and social venues to reopen" (50.3\%), "if I got COVID-19 and recovered from it" (35.7\%), "if sex-venue admission was restricted to those who showed positive test results for COVID-19 [SARSCoV-2] antibodies" (32.5\%), and "if sex-venues decreased the number of people admitted at a given time" $(29.2 \%)$.

Multivariable Associations with Willingness to Attend Sex Venues

Table 1 reports on bivariate analyses between comfort attending sex venues and all variables examined. Participants who took the survey between August 8 and September 23 reported significantly higher levels of comfort going to sex venues than those who did so from May 24 to July $8(p<.001)$. The only statistically
Table 2 Behaviors since the beginning of the COVID-19 pandemic and factors that would increase willingness to attend collective sex venues among 342 survey participants

\begin{tabular}{|c|c|c|}
\hline & & $\%$ \\
\hline \multicolumn{3}{|c|}{ Since March 15,2020 , have you done any of the following? } \\
\hline Had sex with a partner I do not live with & 235 & $(68.7)$ \\
\hline Met new partners online for in-person sex & 196 & $(57.3)$ \\
\hline Had group sex (threesome or more) & 121 & $(35.4)$ \\
\hline Attended a social gathering (party) & 119 & $(34.8)$ \\
\hline \multicolumn{3}{|c|}{$\begin{array}{l}\text { Which of the following would make you more comfortable about } \\
\text { attending a sex venue in the context of the COVID-19 pan- } \\
\text { demic? }\end{array}$} \\
\hline $\begin{array}{l}\text { If a COVID-19/SARS-CoV-2 vaccine was } \\
\text { developed }\end{array}$ & 277 & $(81.0)$ \\
\hline $\begin{array}{l}\text { If the number of new daily infections decreased } \\
\text { significantly }\end{array}$ & 211 & $(61.7)$ \\
\hline $\begin{array}{l}\text { If sex-venue promoters screened for fever before } \\
\text { entry }\end{array}$ & 191 & $(55.8)$ \\
\hline $\begin{array}{l}\text { If I tested positive for COVID-19/SARS-CoV-2 } \\
\text { antibodies }\end{array}$ & 184 & $(53.8)$ \\
\hline $\begin{array}{l}\text { If authorities allowed bars and social venues to } \\
\text { reopen }\end{array}$ & 172 & $(50.3)$ \\
\hline If I got COVID-19 and recovered from it & 122 & $(35.7)$ \\
\hline $\begin{array}{l}\text { If sex-venue promoters restricted entry to people } \\
\text { who showed positive test results for } \\
\text { COVID-19/SARS-CoV-2 antibodies }\end{array}$ & 111 & $(32.5)$ \\
\hline $\begin{array}{l}\text { If sex-venue promoters decreased the number of } \\
\text { people admitted at a given time }\end{array}$ & 100 & $(29.2)$ \\
\hline
\end{tabular}

significant demographic difference was that participants identifying as cisgender men reported a higher level of comfort than those of other sex/gender identities $(p=.027)$. Participants who had gone to sex venues 5 times or more reported a significantly greater level of comfort than those who attended fewer times $(p=.004)$, as well as those who reported using poppers $(p=.017)$, ED drugs $(p=.005)$, or any hard drug at sex venues in the prior year $(p<.001)$. Those who had ever taken a test for SARS-CoV-2 $(p=.004)$ and those who reported having tested positive for it $(p<.001)$ reported significantly greater comfort going to sex venues than those who did not. Finally, participants with lower levels of worry about COVID-19 were significantly more likely to be comfortable attending sex venues than those with higher levels of worry $(p<.001)$.

Multivariable ordinal logistic regression (Table 3) showed that those who completed the survey between August 8 and September 23 (adjusted odds ratio $[\mathrm{aOR}]=2.90, \mathrm{CI}_{95 \%} 1.90$ to 4.43 ), those who attended sex venues at least 5 times in the prior year $(\mathrm{aOR}=1.94$, 
$\mathrm{CI}_{95 \%} 1.26$ to 2.99 ), those who had used hard drugs at sex venues in the prior year $\left(\mathrm{aOR}=1.98, \mathrm{CI}_{95 \%} 1.22\right.$ to 3.23), those who had tested positive for SARS-CoV-2 $\left(\mathrm{aOR}=2.27, \mathrm{CI}_{95 \%} 1.17\right.$ to 4.39$)$, and those with lower levels of worry about COVID-19 (aOR $=3.91, \mathrm{CI}_{95 \%}$ 2.51 to 6.10 ) had higher odds of being comfortable going to sex venues in the context of the COVID-19 pandemic. Although significant in bivariate associations, sex/gender identity and having used poppers or ED drugs at sex venues in the prior year were not significantly associated with the outcome in the multivariable model. We tested the model for all two-way interactions between independent variables and found no interaction to be statistically significant.

\section{Open Survey Answers}

Seventy-nine $(23.1 \%)$ participants wrote an answer to the open question asking them to discuss anything that affected their willingness to attend sex venues or not in the context of the COVID-19 pandemic. Responses included reasons for or against attending sex venues and factors that would increase their willingness to go. The types of responses that were mentioned by at least four participants are presented in Table 4 with representative quotes.

Reasons for or against attending sex venues were generally related to personal risk tolerance and notions of social responsibility. Some participants expressed willingness to tolerate a certain amount of risk by going to sex venues (quote 1), while others felt that attending them seemed simply too risky (quote 5) or irresponsible (quote 4). Indeed, some participants explained their unwillingness to attend on the basis that nothing could be done to minimize the high risk of transmission at sex venues (quote 6). Some participants felt safe attending sex venues because they had already been infected with SARS-CoV-2 and trusted that antibodies would protect them from reinfection (quote 2), but others said that the lack of knowledge regarding the possibility of being reinfected deterred them from attending (quote 7). Some also expressed a sense of pandemic fatigue, feeling that going to sex venues was justified to mitigate the ongoing psychological strain of isolation (quote 3 ). In contrast, other participants would not do anything that could expose them to SARS-CoV-2 to avoid transmitting it to others in their life who might be more vulnerable to its consequences (quote 8).
For many participants, the only factors that would increase their willingness to attend sex venues again were major developments in the control of the COVID-19 pandemic. Some participants mentioned that they would be willing to attend once infection rates decreased substantially or after a certain "wave" of the pandemic (quote 11). Others said they would only be willing to go back to such venues once a vaccine or treatment was available and widely accessible (quotes 9 and 10).

Other participants mentioned prevention strategies that could be adopted at sex venues to make them feel safer about attending during the pandemic. Many participants mentioned the use of some screening measures, for example, requiring a positive SARS-CoV-2 antibody test at entry (quote 12) or doing temperature checks. Another frequently mentioned strategy was ensuring proper ventilation of the venues or hosting outdoor sex parties (quote 13). Many participants also mentioned that all patrons should be required to wear face masks (quote 14) and that the venue staff should take great care cleaning and disinfecting the space properly throughout events (quote 15). Finally, some participants mentioned they would be interested in events that focused on practices with lower risk of SARS-CoV-2 transmission, for example, mutual masturbation, fetish play, or the use of "glory holes" (partitions with holes around the genital area; quote 16). Others said they would be comfortable attending events with smaller numbers of attendees than usual (quote 17).

\section{Discussion}

As NYC was heavily hit by the COVID-19 pandemic beginning in March 2020, public health officials advised residents against hosting or attending collective sex events. This analysis sought to examine how the pandemic impacted SGM individuals who have sex with men in NYC who attend collective sex venues. Among our sample of 342 NYC residents who had attended collective sex venues in the prior year, $9.9 \%$ reported having ever received a positive diagnosis for SARSCoV-2 infection or antibodies. SGM individuals engaging in collective sex might be a population at elevated risk of infection with viruses such as SARS-CoV-2 considering that nearly 1 in 10 participants in this study received a positive test result. However, as this survey did not include a comparison group, we cannot say 
Table 3 Ordinal logistic regressions: willingness to attend sex venues in the context of the COVID-19 pandemic

\begin{tabular}{|c|c|c|c|c|}
\hline \multirow[t]{2}{*}{ Variable } & \multicolumn{2}{|c|}{ Bivariate } & \multicolumn{2}{|c|}{ Multivariable $^{\mathrm{a}}$} \\
\hline & OR & $95 \% \mathrm{CI}$ & $\mathrm{aOR}$ & $95 \% \mathrm{CI}$ \\
\hline Took survey August-September ${ }^{\mathrm{b}}$ & 2.80 & $1.89-4.16^{* * *}$ & 2.90 & $1.90-4.43 * * *$ \\
\hline Cisgender $\operatorname{man}^{\mathrm{c}}$ & 2.16 & $2.16-4.32 *$ & 1.55 & $0.74-3.26$ \\
\hline Attended CSV 5+ times, $\mathrm{PY}^{\mathrm{d}}$ & 1.75 & $1.19-2.58^{* *}$ & 1.94 & $1.26-2.99 * *$ \\
\hline Tested positive for CV $-19^{e}$ & 3.58 & $1.88-6.83^{* * *}$ & 2.27 & $1.17-4.39 *$ \\
\hline Low/no worry about CV $-19^{\text {f }}$ & 4.74 & $3.07-7.31 * * *$ & 3.91 & $2.51-6.10 * * *$ \\
\hline Used poppers at CSV, $\mathrm{PY}^{\mathrm{g}}$ & 1.62 & $1.10-2.40 *$ & 1.25 & $0.82-1.90$ \\
\hline Used EDD at CSV, PY & 1.80 & $1.20-2.70 * *$ & 1.36 & $0.86-2.15$ \\
\hline Used hard drugs CSV, PY & 2.64 & $1.67-4.16^{* * *}$ & 1.98 & $1.22-3.23 * *$ \\
\hline
\end{tabular}

Measured with a Likert scale where $1=$ extremely uncomfortable, $2=$ somewhat uncomfortable, $3=$ neither comfortable nor uncomfortable, $4=$ somewhat comfortable, and $5=$ extremely comfortable

${ }^{\mathrm{a}} n=342$. Model $\chi^{2}=111.995, \mathrm{df}=8, p<0.001$

${ }^{\mathrm{b}}$ Compared to participants who took survey May-July

${ }^{\mathrm{c}}$ Compared to participants who identified as transgender/nonconforming

${ }^{\mathrm{d}}$ Compared to participants who attended CSV 1 to 4 times in the prior year

${ }^{\text {e }}$ Compared to participants who reported never testing positive for CV-19

${ }^{\mathrm{f}}$ Compared to participants who indicated being quite or extremely worried about CV-19

${ }^{g}$ Compared to participants who did not use those substances at CSV in the prior year (hard drugs include GHB, MDMA, special K, cocaine, crystal meth, or LSD)

$C S V$, collective sex venue; $P Y$, past year; $C V$-19, COVID-19/SARS-CoV-2; $E D D$, erectile dysfunction drugs; $O R$, odds ratio; $a O R$, adjusted odds ratio

$* p<.05$

$* * p<.01$

$* * * p<0.001$

whether this high rate of infection is related to engagement in collective sex or to other characteristics of the population. Furthermore, as we did not collect data about the time of diagnosis, it is impossible to know whether these infections resulted from behaviors occurring before or after the beginning of social distancing measures. Nevertheless, considering the high rate of diagnoses among this sample of sex venue attendees, organizers of such venues should proceed with caution when they consider resuming their activities.

Only a minority $(27.5 \%)$ of participants expressed willingness to attend collective sex venues in the context of the COVID-19 pandemic. In multivariable analyses, we found that those who were willing to do so were more likely to have attended sex venues with high frequency in the prior year and more likely to have used hard drugs at these venues. These findings echo those of other studies, which reported that SMM who engaged in group sex or chemsex (sexualized drug use) were, compared to their counterparts, less likely to follow social distancing guidelines or to report a decrease in sexual activity with non-steady partners during the pandemic $[10,11,13,14]$. Future research should examine the underlying reasons why adhering to social distancing guidelines remains difficult for some SGM individuals engaging in collective sex and/or chemsex. Past studies of HIV-related risk behavior among SMM may provide important clues given the associations that have been found between sexual compulsivity, substance use, and engagement in group sex [27-29]. In addition, it is important to note that there are plausible mechanisms through which the use of certain substances (such as stimulants and opioids) may increase biological vulnerability to SARS-CoV-2 infection and COVID-19 progression [30, 31]. In other words, SGM individuals who engage in group sex and use certain substances may be at high risk for SARS-CoV-2 infection and severe COVID-19 symptoms due to both behavioral and 
Table 4 Qualitative analysis of reasons for or against attending sex venues in the context of the COVID-19 pandemic $(n=79)$

Theme $n$ Quotes

Willingness to attend sex venues

Accepting risks $\quad 4$ (1) I'll be glad when they're back. Whatever the public health requires, great, but I figure everyone at a sex party is more comfortable with calculated risks than the general population - and most of us are in the habit of paying close attention to our health as the tradeoff.
Already had
4 (2) I already had COVID-19 and have
COVID-19
antibodies, so I'm not afraid of reinfection myself.
Pandemic fatigue 4 (3) At this point everyone is just feeling caged up a wants and small sense of security to go somewhere to let loose.

Unwillingness

Seems irresponsible/risky

9 (4) Oh I absolutely do not care for sex parties at the moment. I think it's irresponsible and selfish.

(5) To be honest I'd be uncomfortable going to a bar with close friends right now. I don't think I'll be swapping spit with strangers until I confidently feel there is no public health risk.

No prevention effective

5 (6) Reducing the number of patrons allowed doesn't do much for peace of mind since it's not like I'd be social distancing from them be it 5 people or 20.

Reinfection
concerns

8 (7) I don't particularly trust the accuracy of antibody testing and it isn't clear to me that people who have been infected can't be re-infected, so until there's a vaccine, it's very unlikely that I will attend large indoor gatherings like sex parties.

Protecting others 4 (8) I am mostly worried about Covid bc I don't want to give it to someone else who is at risk, like my parents. If I don't expect to see old ppl anytime soon, my tolerance for Covid risk increases.

Factors that would increase willingness

Vaccine

10 (9) Won't go back until there is a safe and effective and widely distributed vaccine

COVID-19

5 (10) I would regard a Covid-19

treatment treatment on par with current HIV

Table 4 (continued)

\begin{tabular}{lcl}
\hline Theme & $n$ Quotes \\
\hline & $\begin{array}{l}\text { Treatment to be equivalent to a } \\
\text { vaccine, in terms of returning to my } \\
\text { previous sexual behavior }\end{array}$ \\
$\begin{array}{c}\text { Reduction in } \\
\text { infection rates }\end{array}$ & $\begin{array}{c}\text { (11) I think I'll wait til the expected } \\
\text { "second wave" comes and goes } \\
\text { before I attend group events. }\end{array}$
\end{tabular}

Strategies to minimize risk at sex venues

Screening

Air circulation and outdoors

Face masks

Cleaning/-

disinfecting

Safer practices

Smaller groups

12 (12) I think it would be insane for a sex venue to open up unless everyone they allow to enter could positively demonstrate with documentation that they had Covid antibodies.

10 (13) Better ventilation seems key--that could help at indoor venues......or an outdoor sex party in a controlled space (private back yard, parking lot, etc) would be very cool!

9 (14) All attendees should be required to wear masks at all times and staff should enforce the requirement and be authorized to tell people that they have to leave if they won't comply.

7 (15) Assurances (and evidence) that the hosts are taking precautions and have implemented a sensible cleaning/sanitizing regiment at the venue.

8 (16) Maybe encouraging lower-contact sexual activities mutual masturbation, gloryholes (those are always hot anyway lol)

4 (17) Sex venues, to me, are potential super spreader locations. it feels a little different than a foursome at a friends house. I feel confident I would be able to track down those people (if I *had* to) after the fact. But a more produced event/party feels more "stranger danger". It also feels like an exponential step up from a smaller house party orgy 
biological factors. Efforts to minimize the transmission of viruses such as SARS-CoV-2 are thus especially important among this population.

Our findings also indicated that pandemic fatigue might be increasing willingness to attend sex venues while the pandemic is ongoing. Participants who completed the survey in August or September expressed significantly greater willingness to attend sex venues than those who did so in May, June, or July 2020. Although we did not collect data longitudinally, another study with SMM found that while participants initially reported reduced sexual activity early in the pandemic, their sexual activity then significantly increased several months later [32]. Furthermore, in our survey's opentext answers, some participants expressed an eagerness to attend sex venues to counter ongoing isolation. Although public health officials should keep emphasizing that avoiding sexual activity with sex partners outside of one's household is an effective way to prevent SARSCoV-2 transmission, they should also acknowledge that doing so will not be achievable for everyone and that some will keep engaging in practices such as collective sex during the pandemic. Indeed, two-thirds of our sample reported having had sex with a partner outside of their household since March 15, 2020, and a third reported having had group sex. Therefore, recommendations on how to minimize the risk of transmission in collective sex settings are urgently needed.

In this study's qualitative data, participants described several strategies that would make them feel safer about attending sex venues in the context of the COVID-19 pandemic. However, some of these strategies would probably be minimally effective. For example, temperature checks are unlikely to screen out individuals who have asymptomatic SARS-CoV-2 infection and who may still transmit the virus [33]. Although outdoor sex parties or the use of face masks might reduce the risk of transmission via respiratory droplets, such measures may have limited effectiveness if attendees are in very close contact with one another, breathe heavily, or exchange saliva. Limiting the types of sexual activities allowed could be a more effective strategy to reduce risk. For instance, the NYC DoHMH recommended [6] that if people choose to have sex with partners outside of their household, they should engage in sexual activities that limit face-to-face contact or use physical barriers (such as walls with openings known as "glory holes"). Because many collective sex events in NYC operated clandestinely even prior to the pandemic, decisions on what strategies should be implemented to reduce the risk of SARS-CoV-2 transmission at sex parties will likely be made by organizers and attendees. Therefore, public health officials should work closely with sex-venue organizers and attendees to find solutions that would be both effective and acceptable to those who want to attend these venues.

Finally, results from this study emphasize the importance of ensuring that sexual health services remain operational and accessible during pandemics such as COVID19. Participants in other studies have reported difficulty accessing HIV/STI testing, PrEP services, or HIV care since the beginning of the pandemic $[32,34]$. Some researchers had hypothesized that there could be a decrease in transmission of HIV and STIs due to social distancing measures [35], but the limited availability of testing and treatment services during the pandemic might also result in an increased number of undiagnosed and/or untreated cases of HIV or other STIs. Although our study did not examine changes in HIV/STI testing or treatment practices, the reports of ongoing sexual activity during the pandemic underscore the need for accessible sexual health services during times of social distancing.

Findings from this study are limited by its reliance on a convenience sample of participants who self-enrolled in the study. Although online recruitment is an effective way to enroll individuals from a hard-to-reach population such as SGM individuals engaged in collective sex, it does not allow for generalization to the larger population [36]. Furthermore, as the main venues for recruitment were hookup apps (smartphone applications used to meet sex partners) and emails sent by sex-venue organizers, this study might have overlooked the perspective of individuals who were not using/consulting these media during the COVID-19 pandemic. For example, it is possible that individuals who use hookup apps or read emails related to sex parties during the pandemic are more willing to attend sex venues than those who do not. Also, individual sex-venue organizers often have a specific clientele, and thus, the results might be more representative of the clientele of the promoters who agreed to collaborate with the study team by sending recruitment advertisements. Another limitation is that data were self-reported; therefore, results about SARS-CoV-2 diagnoses should not be regarded as actual incidence or prevalence among the sample or population. Future studies should use more reliable measures of SARS-CoV-2 infection such as reverse transcriptase-polymerase chain reaction (RT-PCR) and/or antibody test results. Social desirability bias might 
also have influenced some responses, although it is generally minimal in confidential, self-administered online surveys [37]. Nevertheless, some participants might have been reluctant to express opinions or report behaviors that they thought might be viewed negatively.

In conclusion, individuals attending collective sex venues could be at elevated risk for infection with viruses such as SARS-CoV-2, and these venues might be important sites of transmission during pandemics like COVID19. Although only a minority of SGM participants in our study expressed being comfortable attending collective sex venues in the context of the COVID-19 pandemic, some would be willing to attend with the use of various risk reduction strategies. Public health authorities should offer guidelines or resources to help minimize risk in such environments during pandemics such as COVID-19.

Acknowledgments This study was supported by a subaward (to Étienne Meunier) from the Fordham HIV and Drug Abuse Prevention Research Ethics Training Institute (RETI), a training grant sponsored by the National Institute on Drug Abuse (R25DA031608, PI: Celia B. Fisher).

\section{References}

1. Goldstein J, McKinley J. Coronavirus in N.Y.: Manhattan Woman Is First Confirmed Case in State. The New York Times. https://www.nytimes.com/2020/03/01 /nyregion/new-york-coronvirus-confirmed.html. Published March 2, 2020. Accessed 20 Dec 2020.

2. Woodward SN Aylin. A comprehensive timeline of the coronavirus pandemic at 1 year, from China's first case to the present. Business Insider. Published online December 15, 2020. Accessed 20 Dec 2020. https://www. businessinsider.com/coronavirus-pandemic-timelinehistory-major-events-2020-3

3. Kerr A. Timeline: How COVID-19 took over NYC. Investopedia. Published online October 6, 2020. Accessed 20 Dec 2020. https://www.investopedia.com/historical-timelineof-covid-19-in-new-york-city-5071986

4. Pereira I. NYC mayor signals another shutdown coming as COVID-19 cases rise. ABC News. Published online December 14, 2020. Accessed 18 Dec 2020. https://abcnews.go.com/US/nyc-mayor-signals-shutdowncoming-covid-19-cases/story?id=74721084

5. Cipriano M, Giacalone A, Ruberti E. Sexual behaviors during COVID-19: the potential risk of transmission. Arch Sex Behav. 2020;49(5):1431-2. https://doi.org/10.1007/s10508020-01757-0.

6. New York City Department of Health and Mental Hygiene. Safer Sex and COVID-19. Published online 2020. Accessed January 9, 2021 . https:// w w 1 . ny c. gov/assets/doh/downloads/pdf/imm/covid-sex-guidance. pdf?utm_source=morning_brew

7. Brennan DJ, Card KG, Collict D, Jollimore J, Lachowsky NJ. How might social distancing impact gay, bisexual, queer, trans and two-Spirit men in Canada? AIDS Behav. 2020;24(9):2480 2. https://doi.org/10.1007/s10461-020-02891-5.

8. Döring N. How is the COVID-19 pandemic affecting our sexualities? An overview of the current media narratives and research hypotheses. Arch Sex Behav. 2020;49(8):2765-78. https://doi.org/10.1007/s10508-020-01790-z.

9. Hammoud MA, Maher L, Holt M, Degenhardt L, Jin F, Murphy D, et al. Physical distancing due to COVID-19 disrupts sexual behaviors among gay and bisexual men in Australia: implications for trends in HIV and other sexually transmissible infections. JAIDS Journal of Acquired Immune Deficiency Syndromes. 2020;85(3):309-15. https://doi.org/10.1097/QAI.0000000000002462.

10. Reyniers T, Rotsaert A, Thunissen E, Buffel V, Masquillier C, van Landeghem E, et al. Reduced sexual contacts with non-steady partners and less PrEP use among MSM in Belgium during the first weeks of the COVID-19 lockdown: results of an online survey. Sex Transm Infect. Published online November 10. 2020; https://doi.org/10.1136 /sextrans-2020-054756.

11. Starks TJ, Jones SS, Sauermilch D, et al. Evaluating the impact of COVID-19: a cohort comparison study of drug use and risky sexual behavior among sexual minority men in the U.S.A. Drug and Alcohol Dependence. 2020;216: 108260. https://doi.org/10.1016/j.drugalcdep.2020.108260

12. van Bilsen WPH, Zimmermann HML, Boyd A, Coyer L, van der Hoek L, Kootstra NA, Hoornenborg E, Prins M, Schim van der Loeff MF, Davidovich U, Matser A. Sexual behavior and its determinants during COVID-19 restrictions among men who have sex with men in Amsterdam. J Acquir Immune Defic Syndr. Published online November 18, 2020. https://doi.org/10.1097/QAI.0000000000002581

13. de Sousa AFL, Oliveira L, Queiroz A, et al. Casual sex among MSM during the period of sheltering in place to prevent the spread of COVID-19: results of national, online surveys in Brazil and Portugal. medRxiv. Published online June 9, 2020. https://doi.org/10.1101/2020.06.07.20113142

14. Stephenson R, Chavanduka TMD, Rosso MT, Sullivan SP, Pitter RA, Hunter AS, et al. Sex in the time of COVID-19: results of an online survey of gay, bisexual and other men who have sex with men's experience of sex and HIV prevention during the US COVID-19 epidemic. AIDS Behav Published online September. 2020;2:40-8. https://doi. org/10.1007/s10461-020-03024-8.

15. Grov C. HIV risk and substance use in men who have sex with men surveyed in bathhouses, bars/clubs, and on Craigslist.org: venue of recruitment matters. AIDS Behav. 2012;16(4):807-17. https://doi.org/10.1007/s10461-011-9999-6.

16. Mimiaga MJ, Reisner SL, Bland S, Cranston K, Isenberg D, Driscoll MA, et al. "It's a quick way to get what you want": a formative exploration of HIV risk among urban Massachusetts men who have sex with men who attend sex parties. AIDS Patient Care \& STDs. 2010;24(10):659-74. https://doi.org/10.1089/apc.2010.0071.

17. Phillips G, Grov C, Mustanski B. Engagement in group sex among geosocial networking mobile application-using men 
who have sex with men. Sex Health. 2015;12(6):495-500. https://oi.org/10.1071/SH15108.

18. Prestage GP, Hudson J, Down I, Bradley J, Corrigan N, Hurley $M$, et al. Gay men who engage in group sex are at increased risk of HIV infection and onward transmission. AIDS Behav. 2009;13(4):724-30. https://doi.org/10.1007 /s10461-008-9460-7.

19. Balán IC, Barreda V, Marone R, Avila MM, CarballoDiéguez A. Venues, patrons, and alcohol use dynamics: the creation of a high risk sexual environment. AIDS Behav. 2014;18(11):2097-109. https://doi.org/10.1007 /s10461-014-0759-2.

20. Friedman SR, Mateu-Gelabert P, Sandoval M. Group sex events amongst non-gay drug users: an understudied risk environment. Int J Drug Policy. 2011;22(1):1-8. https://doi. org/10.1016/j.drugpo.2010.06.004.

21. Meunier É. No attitude, no standing around: the organization of social and sexual interaction at a gay male private sex party in New York City. Arch Sex Behav. 2014;43(4):68595. https://doi.org/10.1007/s10508-013-0182-1.

22. New York State. Public Health Law.; 2000. Accessed 19 May 2012. https://regs.health.ny.gov/content/subpart-24-2prohibited-facilities

23. Vines B. Lessons of AIDS in mind as queer sex party industry reacts to coronavirus. City Limits. Published March 17, 2020. Accessed 30 Dec 2020. https://citylimits. org/2020/03/17/lessons-of-aids-in-mind-as-queer-sex-partyindustry-reacts-to-coronavirus/

24. Baume M. NYC Gays Threw a Coronavirus Dance Party, and People are Furious. them. Published May 7, 2020. Accessed 30 Dec 2020. https://www.them.us/story/nycgays-threw-a-coronavirus-dance-party-and-people-arefurious

25. Closson T. Party at a Queens Sex Club With 80 People Is Shut Down by Sheriff. The New York Times. https://www. nytimes.com/2020/11/22/nyregion/sex-club-covid-sheriffnyc.html. Published November 23, 2020. Accessed 30 Dec 2020.

26. Colyar B. New York nightlife never stopped. It just moved underground. The Cut. Published November 23, 2020. Accessed 30 Dec 2020. https://www.thecut.com/2020/11 /nyc-underground-nightlife-covid-19.html

27. Woolf-King SE, Rice TM, Truong H-HM, Woods WJ, Jerome RC, Carrico AW. Substance use and HIV risk behavior among men who have sex with men: the role of sexual compulsivity. J Urban Health. 2013;90(5):948-52. https://doi.org/10.1007/s11524-013-9820-0.

28. Grov C, Parsons JT, Bimbi DS. Sexual compulsivity and sexual risk in gay and bisexual men. Arch Sex Behav. 2010;39(4):9409. https://doi.org/10.1007/s10508-009-9483-9.
29. Carrico AW, Pollack LM, Stall RD, Shade SB, Neilands TB, Rice TM, et al. Psychological processes and stimulant use among men who have sex with men. Drug Alcohol Depend. 2012;123(1-3):79-83. https://doi.org/10.1016/j. drugalcdep.2011.10.020.

30. Carrico AW, Horvath KJ, Grov C, Moskowitz JT, Pahwa S, Pallikkuth S, et al. Double jeopardy: methamphetamine use and HIV as risk factors for COVID-19. AIDS Behav. 2020;24(11): 3020-3. https://doi.org/10.1007/s10461-020-02854-w.

31. Schimmel J, Manini AF. Opioid use disorder and COVID19: biological plausibility for worsened outcomes. Substance Use \& Misuse. 2020;55(11):1900-1. https://doi. org/10.1080/10826084.2020.1791184.

32. Pampati S, Emrick K, Siegler AJ, Jones J. Changes in sexual behavior, PrEP adherence, and access to sexual health services due to the COVID-19 pandemic among a cohort of PrEP-using MSM in the South. medRxiv. Published online November 12, 2020. https://doi.org/10.1101 /2020.11.09.20228494

33. Sayampanathan AA, Heng CS, Pin PH, Pang J, Leong TY, Lee VJ. Infectivity of asymptomatic versus symptomatic COVID-19. Lancet. 2021;397(10269):93-4. https://doi. org/10.1016/S0140-6736(20)32651-9.

34. Sanchez TH, Zlotorzynska M, Rai M, Baral SD. Characterizing the impact of COVID-19 on men who have sex with men across the United States in April, 2020. AIDS Behav. 2020;24(7):2024-32. https://doi.org/10.1007 /s10461-020-02894-2.

35. Alpalhão $\mathrm{M}$, Filipe $\mathrm{P}$. The impacts of isolation measures against SARS-CoV-2 infection on sexual health. AIDS Behav. 2020;24(8):2258-9. https://doi.org/10.1007/s10461020-02853-x.

36. Grov C, Westmoreland D, Rendina HJ, Nash D. Seeing is believing? Unique capabilities of imen who have sex with men: a review of studies and methodological considerations. JAIDS Journal of Acquired Immune Deficiency Syndromes. 2019;82:S253-60. https://doi.org/10.1097 /QAI.0000000000002217.

37. Grov C, Breslow AS, Newcomb ME, Rosenberger JG, Bauermeister JA. Gay and bisexual men's use of the internet: research from the 1990s through 2013. J Sex Res. 2014;51(4):390-409. https://doi.org/10.1080 /00224499.2013.871626.

Publisher's Note Springer Nature remains neutral with regard to jurisdictional claims in published maps and institutional affiliations. 